\title{
MESOSCOPIC MODELLING OF MASONRY USING GFEM: A COMPARISON OF STRONG AND WEAK DISCONTINUITY MODELS
}

\author{
B. Vandoren ${ }^{1,2}$, K. De Proft ${ }^{2}$ \\ ${ }^{1}$ MoBuild Research Group, XIOS University College (bram.vandoren@xios.be) \\ ${ }^{2}$ Physics Capacity Group, Hasselt University
}

\begin{abstract}
Two mesoscopic masonry models are presented in which the mortar joints are incorporated using the Generalised Finite Element Method (GFEM): a model in which the joints are represented as strong discontinuities and a model in which weak discontinuities are used to model the joints. The bricks remain linear elastic throughout the calculation. The presented models are compared using equivalent damage models through shear wall and settlement tests. The simulations are validated with experimental results available in literature.
\end{abstract}

Keywords: Masonry, GFEM, Mesoscopic models.

\section{INTRODUCTION}

Masonry structures are characterised by their low material cost and relatively simple way of constructing. The computational modelling of these structures remains, however, a great challenge. Two major modelling groups exist: macroscale and mesoscale models [5]. The former approach homogenises the masonry constituents, i.e. the bricks and joints, to one orthotropic material whereas the latter approach models the constituents as separate entities. In this contribution, two mesoscopic masonry models are presented and compared: a model in which the joints are represented as strong discontinuities [2] and a model in which weak discontinuities are used to model the joints [11].

Unlike classical models, where the strong and weak discontinuities are modelled, respectively, by interface [1] and continuum elements [6], the discontinuities are incorporated using the Generalised Finite Element Method [10, 12], whose formulation is described in Section 2. This method allows an in-element embedment of the discontinuities, i.e. the finite element mesh does not need to conform to the masonry joints. The enhanced degrees of freedom, stemming from this method, describe the joint behaviour. As the masonry joints can be seen as a predefined failure pattern, these degrees of freedom are all active at the beginning of the calculation.

As will be shown in Section 3, any continuum material model can be employed to describe the joint behaviour in the weak discontinuity model. This is in contrast with the strong discontinuity approach, in which the constitutive relations are expressed in degenerated stress and strain spaces. An equivalent degenerated constitutive model is therefore presented, in order to make a meaningful comparison between both approaches. The results of this comparative analysis are given in Section 4 through shear wall and settlement tests. 


\section{MODELLING DISCONTINUITIES USING GFEM}

In the developed two-dimensional mesoscopic masonry model, the GFEM technique is used so that the behaviour of each brick is governed by a designated set of enhanced degrees of freedom [12]. Consequently, the displacement field can be decomposed according to

$$
\mathbf{u}=\hat{\mathbf{u}}+\sum_{i=1}^{N_{\mathcal{B}}} \mathcal{H}_{i} \tilde{\mathbf{u}}_{i}
$$

where $N_{\mathcal{B}}$ denotes the total number of bricks within the masonry wall, $\hat{\mathbf{u}}$ is the regular displacement field, $\tilde{\mathbf{u}}_{i}$ and $\mathcal{H}_{i}$ are the enhanced displacement field and enrichment function associated with brick $\mathcal{B}_{i}$, respectively.

In case of the weak discontinuity model, Figure 1(a), a unit ramp function is used [7, 11] whereas the Heaviside step function is used in the strong discontinuity model [2], Figure 1(b). Figure 1 also shows that for the same mesh size and when modelling stack bond masonry, i.e. the joints can be aligned with the element borders, the strong discontinuity model is computationally more attractive than the weak discontinuity model, since only half of the enhanced degrees of freedom is needed. For a more detailed discussion concerning kinematic descriptions and implementation aspects of both models, the reader is referred to [12].

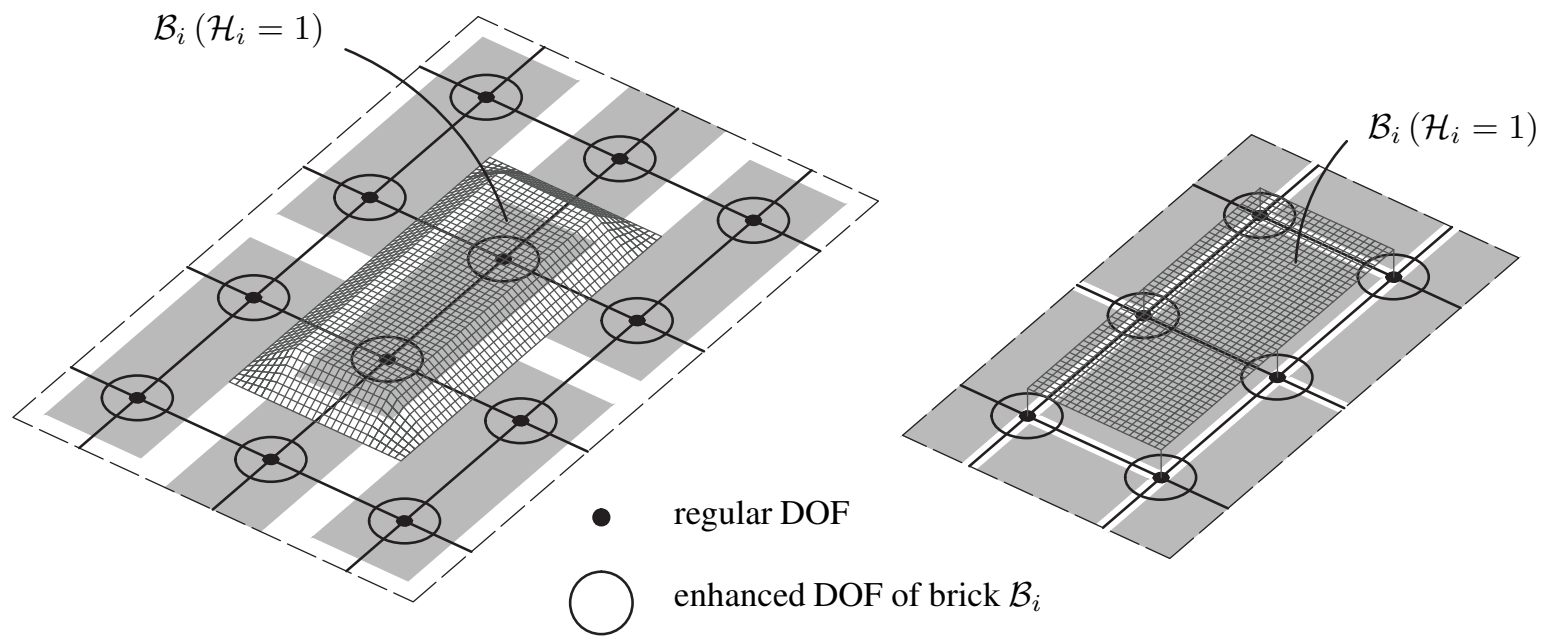

(a) weak discontinuity model

(b) strong discontinuity model

Figure 1. Enrichment functions for a brick $\mathcal{B}_{i}$ : (a) weak discontinuity model (unit ramp function); (b) strong discontinuity model (Heaviside function). 


\section{MATERIAL MODELS}

\subsection{Weak discontinuity model}

In the presented masonry model, the brick behaviour remains linear elastic throughout the simulations, whereas the nonlinear mortar joint behaviour is governed by an isotropic damage law of the form

$$
\boldsymbol{\sigma}=(1-\omega) \mathbf{C}_{w} \varepsilon
$$

in which $\omega$ denotes a scalar damage parameter describing the softening behaviour [4],

$$
\omega=\left\{\begin{array}{ll}
0 & \text { if } \kappa<\kappa_{0} \\
1-\frac{\kappa_{0}}{\kappa} \exp \left[-\frac{\kappa-\kappa_{0}}{\frac{G_{f_{I}}}{f_{t}}-\frac{1}{2} \kappa_{0}}\right] & \text { if } \quad \kappa \geq \kappa_{0}
\end{array},\right.
$$

where $G_{f_{I}}$ denotes the mode I fracture energy and $f_{t}$ is the uniaxial tensile strength of the mortar joints. The threshold upon which damage of the mortar joints initiates is given by

$$
\kappa_{0}=\frac{f_{t}}{E_{m}}
$$

with $E_{m}$ the Young's modulus of the mortar joints. The history parameter $\kappa$ stores the largest value ever attained of the equivalent strain $\varepsilon_{e q}$. The latter is defined through a Drucker-Prager damage model, expressed in terms of strain invariants (assuming plane stress conditions),

$$
\varepsilon_{e q}=\left\{\begin{array}{l}
A \frac{I_{1, \varepsilon}}{1-2 \nu_{m}}+B \frac{\sqrt{J_{2, \varepsilon}}}{1+\nu_{m}} \quad \text { if } \quad \frac{\sqrt{J_{2, \varepsilon}}}{1+\nu_{m}} \geq \frac{A-C}{D-B} \frac{I_{1, \varepsilon}}{1-2 \nu_{m}} \\
C \frac{I_{1, \varepsilon}}{1-2 \nu_{m}}+D \frac{\sqrt{J_{2, \varepsilon}}}{1+\nu_{m}} \quad \text { if } \quad \frac{\sqrt{J_{2, \varepsilon}}}{1+\nu_{m}} \leq \frac{A-C}{D-B} \frac{I_{1, \varepsilon}}{1-2 \nu_{m}}
\end{array}\right.
$$

where $\nu_{m}$ denotes the Poisson's ratio of the mortar joints. The material parameters $A$ and $B$ are calculated by fitting the model to the uniaxial tensile $\left(f_{t}\right)$ and compressive strengths $\left(f_{c}\right)$ [6], whereas $C$ and $D$ define the compressive cap:

$$
\begin{gathered}
A=\frac{1}{2} \frac{f_{c}-f_{t}}{f_{c}}, \\
B=\frac{\sqrt{3}}{2} \frac{f_{c}+f_{t}}{f_{c}}, \\
C=\frac{\left(f_{b}-f_{c}\right) f_{t}}{f_{b} f_{c}}, \\
D=\sqrt{3} \frac{\left(2 f_{b}+f_{c}\right) f_{t}}{f_{b} f_{c}} .
\end{gathered}
$$




\subsection{Strong discontinuity model}

Since the behaviour of the strong discontinuity is expressed in terms of tractions and jumps, the material model needs to be defined in traction (or jump) space. As in the weak discontinuity model, the strong discontinuity model is also damage-driven through

$$
\mathbf{t}_{d}=(1-\omega) \mathbf{T}_{d} \llbracket \mathbf{u} \rrbracket
$$

where $\llbracket \mathbf{u} \rrbracket$ represents the displacement jump at the mortar joint, and $\omega$ is defined by

$$
\omega=\left\{\begin{array}{ll}
0 & \text { if } \kappa<\kappa_{0} \\
1-\frac{\kappa_{0}}{\kappa} \exp \left[-\frac{\kappa-\kappa_{0}}{\frac{h G_{f_{I}}}{f_{t}}-\frac{1}{2} \kappa_{0}}\right] & \text { if } \quad \kappa \geq \kappa_{0}
\end{array},\right.
$$

where $h$ denotes the joint thickness. The components of the material stiffness matrix $\mathbf{T}_{d}=$ $\operatorname{diag}\left(k_{n}, k_{t}\right)$ are calculated according to [9]

$$
\begin{aligned}
k_{n} & =\frac{E_{b} E_{m}}{h\left(E_{b}-E_{m}\right)}, \\
k_{t} & =\frac{G_{b} G_{m}}{h\left(G_{b}-G_{m}\right)}
\end{aligned}
$$

in which $E_{b}$ is the Young's modulus of the bricks. $G_{b}$ and $G_{m}$ are the shear moduli of the bricks and mortar joints, respectively. The damage threshold $\kappa_{0}$ in (11) is taken

$$
\kappa_{0}=\frac{f_{t}}{k_{n}}
$$

The history parameter $\kappa$ stores the largest value ever attained of the equivalent displacement jump $\varepsilon_{e q}$, which is defined by a degenerated Drucker-Prager model, given by

$$
\llbracket \mathrm{u} \rrbracket_{e q}=\left\{\begin{array}{lll}
A I_{\llbracket \mathrm{u} \rrbracket}+B J_{\llbracket \mathrm{u} \rrbracket} & \text { if } \quad J_{\llbracket \mathrm{u} \rrbracket} \geq \frac{A-C}{D-B} I_{\llbracket \mathrm{u} \rrbracket} \\
C I_{\llbracket \mathrm{u} \rrbracket}+D J_{\llbracket \mathrm{u} \rrbracket} & \text { if } \quad J_{\llbracket \mathrm{u} \rrbracket} \leq \frac{A-C}{D-B} I_{\llbracket \mathrm{u} \rrbracket}
\end{array}\right.
$$

in which the variables containing the jump terms are calculated by

$$
\begin{gathered}
I_{\llbracket \mathrm{u} \rrbracket}=\left(1+\nu_{m}\right) \llbracket \mathrm{u}_{\mathrm{n}} \rrbracket \\
J_{\llbracket \mathrm{u} \rrbracket}=\frac{1}{1+\nu_{m}} \sqrt{\frac{1}{6} \llbracket \mathrm{u}_{\mathrm{n}} \rrbracket^{2}\left(1+\frac{1+\nu_{m}^{2}}{\left(1-\nu_{m}\right)^{2}}\right)\left(1-\nu_{m}^{2}\right)^{2}+\frac{1}{4} \llbracket \mathrm{u}_{\mathrm{t}} \rrbracket^{2}} .
\end{gathered}
$$




\section{NUMERICAL EXAMPLES}

\subsection{Shear wall test}

The first example is a shear wall with opening [8]. Tables 1 and 2 list the material parameters. A confining stress of $0,30 \mathrm{~N} / \mathrm{mm}^{2}$ is applied on top of the wall, prior to the application of the horizontal loading. The boundary conditions and test setup are depicted in Figure 2. Figure 3(a) shows the background mesh used in combination with the weak discontinuity model. In case of the strong discontinuity model, the number of finite elements per half brick equals $4 \times 4$.

Table 1. Setup and elastic material parameters for the shear wall test.

\begin{tabular}{cccc}
\hline & dimensions & $E\left[\mathrm{~N} / \mathrm{mm}^{2}\right]$ & $\nu$ \\
\hline joints & $10 \mathrm{~mm}$ & 782 & 0,14 \\
bricks & $52 \times 210 \times 100 \mathrm{~mm}^{3}$ & 16700 & 0,15 \\
\hline
\end{tabular}

Table 2. Inelastic material parameters for the shear wall test.

\begin{tabular}{ccccc}
\hline & $f_{t}\left[\mathrm{~N} / \mathrm{mm}^{2}\right]$ & $f_{c}\left[\mathrm{~N} / \mathrm{mm}^{2}\right]$ & $f_{b}\left[\mathrm{~N} / \mathrm{mm}^{2}\right]$ & $G_{f_{I}}[\mathrm{~N} / \mathrm{mm}]$ \\
\hline joints & 0,25 & 10,5 & $1,2 f_{c}$ & 0,018 \\
\hline
\end{tabular}

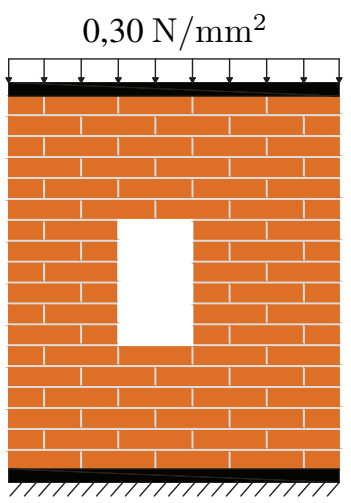

(a)

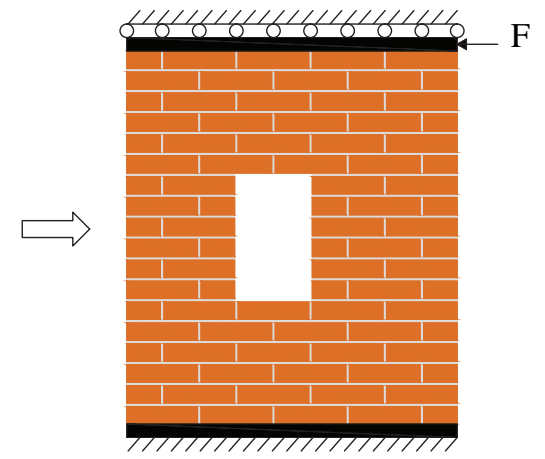

(b)

Figure 2. Test setup and boundary conditions for the shear wall test:

(a) phase 1: confining load; (b) phase 2: horizontal loading.

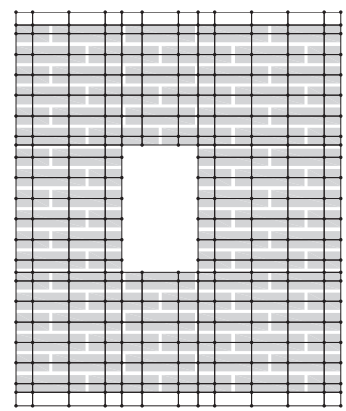

(a)

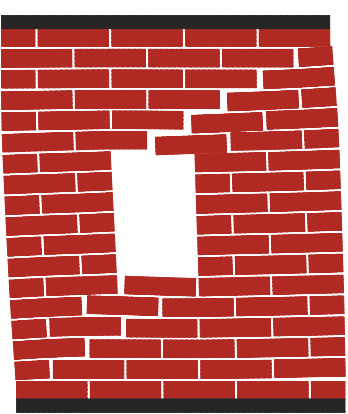

(b) weak discontinuity model

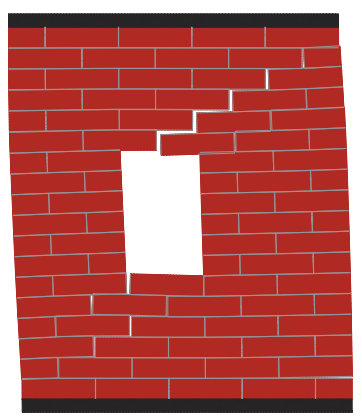

(c) strong discontinuity model

Figure 3. Background mesh (a) and deformed meshes (b-c) for the shear wall tests at a loading point displacement (LPD) of 4,0 $\mathrm{mm}$. The deformations are magnified by 10 . 
The results of all models show a good agreement with those from the experiments. The typical staircase crack patterns, observed in the experiments, are obtained (Figure 3(b-c)) and all simulated peak loads are situated between the observed peak loads (Figure 4).

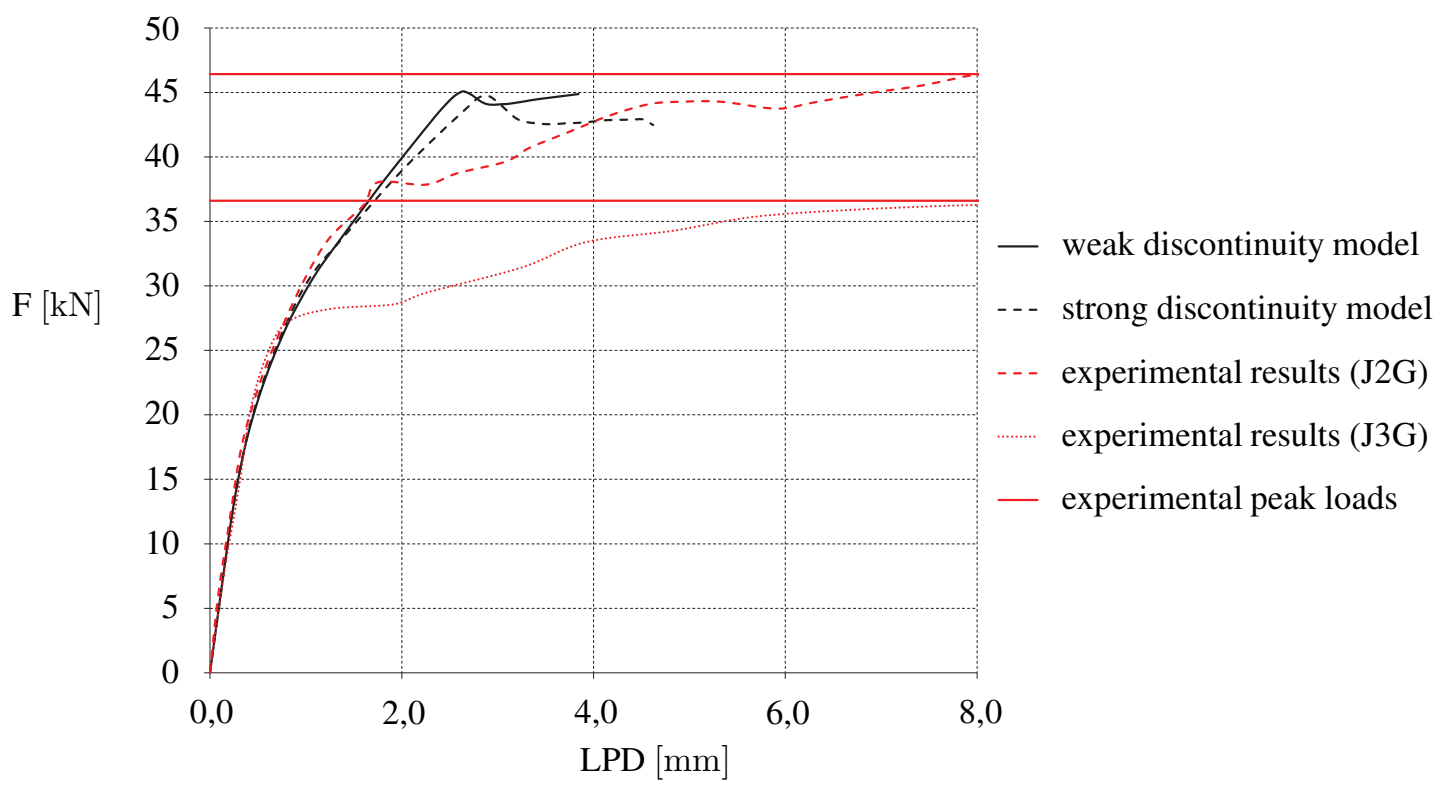

Figure 4. Shear wall test: comparative results of both weak and strong discontinuity models.

\subsection{Settlement test}

In this final example, a blind wall is subjected to an end-settlement. The brick dimensions equal $190 \times 290 \times 140 \mathrm{~mm}^{3}$, whereas the elastic and inelastic material properties are those from the previous simulation (Tables 1 and 2). The behaviour of the soil is governed by an interface element (indicated in blue in Figures 5 and 6). Again, both masonry models are able to predict realistic failure modes when compared to experimental data [3].

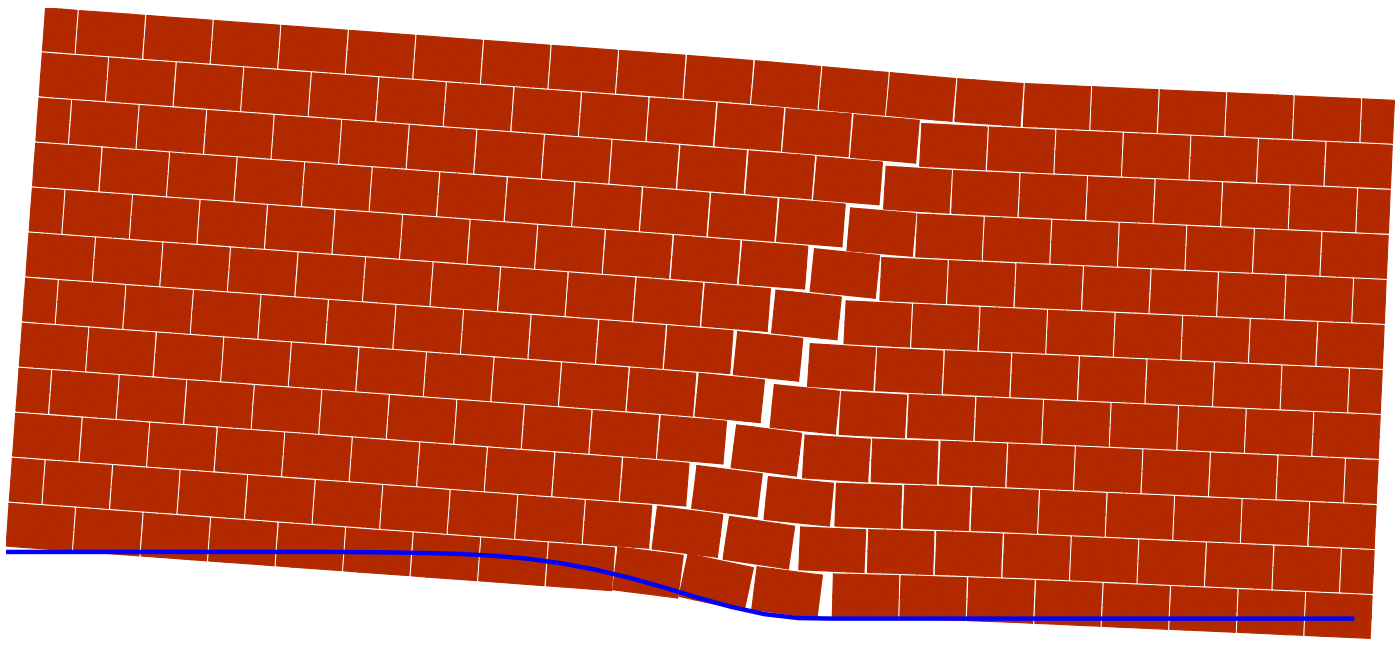

Figure 5. Settlement test: weak discontinuity model. 


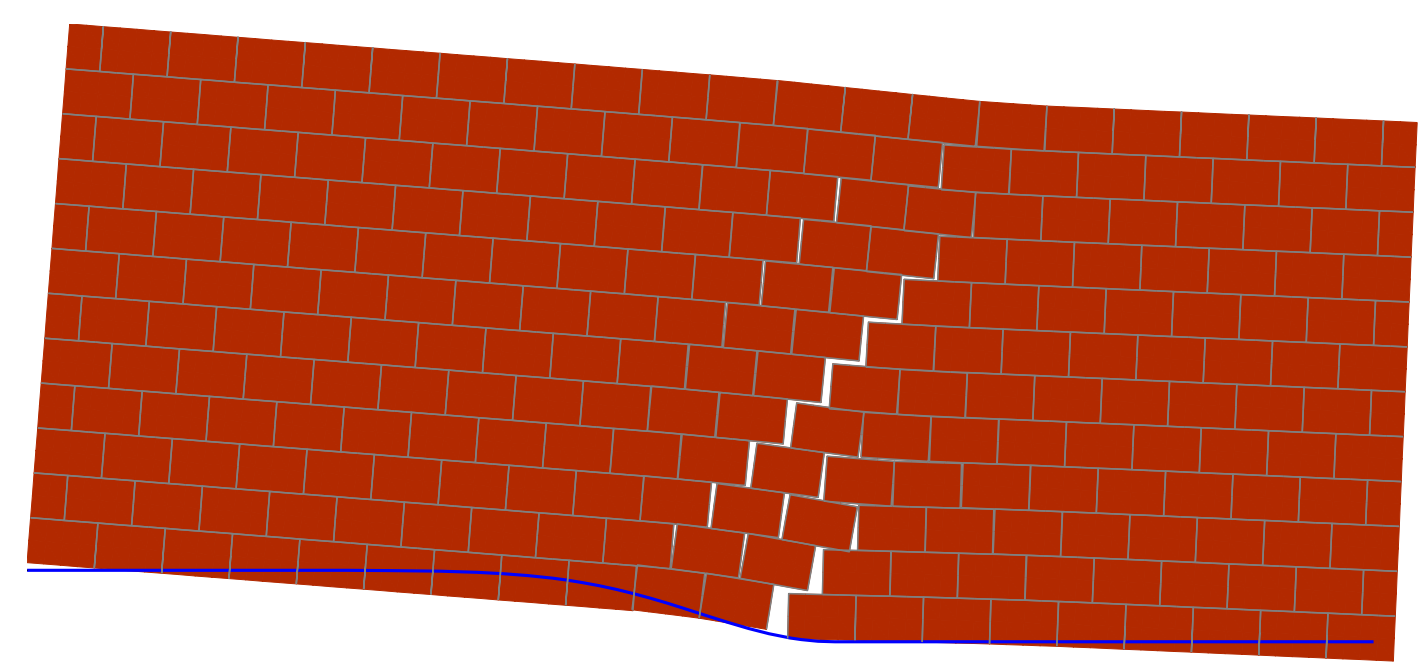

Figure 6. Settlement test: strong discontinuity model.

\section{CONCLUSIONS}

In this contribution, two mesoscopic masonry models are presented and compared in which joints are modelled by weak and strong discontinuities. The discontinuities are element-embedded using the Genaralised Finite Element Method. The main difference between both models is that in case of the weak discontinuity model, continuum material models can be employed to describe the joint behaviour. The strong discontinuity model requires a degenerated material model, expressed in traction space. A comparative analysis was made between both models through shear wall and settlement tests using a continuum and degenerated Drucker-Prager material model. It was shown that both weak and strong discontinuity models are capable of predicting realistic crack patterns and peak loads.

\section{Acknowledgements}

The support of this research by the Bijzonder Onderzoeksfonds Doctoral Funding program of Hasselt University (BOF-DOC) is gratefully acknowledged. Furthermore, both authors wish to thank Professor Bert Sluys and Assistent Professor Angelo Simone of the Computational Mechanics Group at Delft University of Technology for their helpful comments and suggestions. 


\section{REFERENCES}

[1] Alfaiate J. V., de Almeida J. R., "Modelling discrete cracking on masonry walls". Masonry International 17(2), 83-93, 2004.

[2] De Proft K., Heyens K., Sluys L. J., "Mesoscopic modelling of masonry failure". Proceedings of the ICE - Engineering and Computational Mechanics 164(EM1), 41-46, 2010.

[3] de Vent, I., "Structural damage in masonry: Developing diagnostic decision support". PhD thesis, TU Delft, 2012.

[4] Feenstra P. H., "Implementing an isotropic damage model in Diana. Use-case for the user-supplied subroutine usrmat". Proceedings of the Third DIANA World Conference, 89-97, 2002.

[5] Lourenço P. B., Rots J. G., Blaauwendraad J., "Two approaches for the analysis of masonry structures". HERON 40(4), 313-340, 1995.

[6] Massart T. J., Peerlings R. H. J., Geers M. G. D., "Mesoscopic modeling of failure and damage-induced anisotropy in brick masonry". European Journal of Mechanics a-Solids 23(5), 719-735, 2004.

[7] Oliver J., Cervera M., Manzoli O., "Strong discontinuities and continuum plasticity models: the strong discontinuity approach”. Int. J. Plasticity 15(3), 319-351, 1999.

[8] Raijkmakers T. M. J., Vermeltvoort A. T., "Deformation controlled tests in masonry shear walls - Report B-92-1156”. TNO Bouw, 1992.

[9] Rots J. G., "Structural masonry: An experimental/numerical basis for practical design rules (CUR Report 171)". Civieltechnisch Centrum Uitvoering Research en Regelgeving, 1997.

[10] Simone A., Duarte C. A., Van der Giessen E., "A Generalized Finite Element Method for polycrystals with discontinuous grain boundaries". Int. J. Numer. Meth. Engng 67(8), 1122-1145, 2006.

[11] Vandoren B., De Proft K., "Mesoscopic modelling of masonry using embedded weak discontinuities based on partitions of unity". Proceedings of the 2nd International Conference on Extended Finite Element Methods (XFEM 2011) 1, 72-75, 2011.

[12] Vandoren B., De Proft K., Simone A., Sluys L. J., "Mesoscopic modelling of masonry using weak and strong discontinuities". Manuscript submitted for publication, 2012. 